\title{
Automated, web-based environment for daily fire risk assessment in New Caledonia
}

\author{
G. Wattelez $^{a}$, Touraivane ${ }^{a}$, M. Mangeas ${ }^{b}$, J. André $^{b}$ and A. Couturier ${ }^{a}$ \\ ${ }^{a}$ University of New Caledonia, ${ }^{b}$ Institut de Recherche pour le Développement \\ Email: guillaume.wattelez@univ-nc.nc
}

\begin{abstract}
In New Caledonia, the increasing number, frequency, and extent of fires represent both a danger to human life and a threat to ecosystems conservation in an area considered to be a sanctuary of biodiversity.

A four-year study was carried out in the framework of the INC research project to better understand why fires start and to improve fire prevention. Based on satellite observations, a model was built to calculate the risk of fire ignition, together with a Bayesian network to link data on social, environmental, and climatological risk factors. The model and environmental, geological, and topographical data can be used to assess the impacts of fire on biodiversity and erosion.

The purpose of this paper is to present a tool designed to produce on-the-fly maps showing fire risk obtained. This web-based tool implements the model built during the research project and provides fire risk maps every day thanks to an automated recovery of climate data. The user only has to select the date of interest in order that the tool manages all the process of maps creation and display.

Open Geospatial Consortium (OGC) is an international organization in which governments, trade research, and non-profit structures collaborate to implement open standards related to services and geospatial content, as well as GIS data processing and sharing. The presented tool has been made with some standards specified by OGC (Geography Markup Language, Web Map Service), allowing it to be interoperable with other systems that implements OGC standards.

An online tool that exploits live results of the model and automated filling of a database enables identification of high fire risk sites. It then facilitates the task of land and environmental management by combining geographic data on location of water resources and roads that can be used to reach the potentially dangerous sites of fires.
\end{abstract}

Keywords: Fire risk assessment, statistical model, interoperability, dynamic maps, OGC (Open Geospatial Consortium) 


\section{INTRODUCTION}

In New Caledonia, the number of fires, their frequency and their extent are increasing. This represents not only a danger to human life but also threatens the conservation of ecosystems that are considered to be a world hotspot of biodiversity. Every year, tens of thousands of hectares of forest go up in smoke. This critical situation reached a peak in 2005 when a fire destroyed the Montagne des Sources in the town of Mont-Dore and spread to the town of Dumbéa. The fire spread very rapidly due to adverse weather conditions (wind and heat) as well as a lack of fire fighting equipment and human resources. In seven days, 4,300 hectares of land were burned. This is all the more catastrophic as the damage occurred in areas considered as ecological treasures. Indeed, Caledonian forests are host to unique plant and animal species in the world.

The WWF (World Wide Fund for Nature), which was strongly committed to fighting this fire, asked researchers to look for ways to avoid the same kind of disaster happening in the future. With funding from the French Research Agency (Agence Nationale de la Recherche, ANR), a four-year study was carried out in the framework of the INC (Incendies et biodiversité des écosystèmes en Nouvelle-Calédonie) project, involving 40 scientists from different scientific institutions in four major fields (fire regime and propagation, ecosystems and biodiversity, human and social practices, and meteorology and climate). An unprecedented amount of information was collected. It included satellite images, a fire chronicle, local knowledge (ancient and contemporary) from indigenous peoples. Its analysis helped understand the behaviour of fires in New Caledonia.

Today provided the availability of data, a daily ignition risk can be computed, and the impacts of a fire on biodiversity and soil erosion can be calculated. Here we describe a web-based application that highlights the study about fires in New Caledonia because model results are now available to the general public. This is a user-friendly application that makes it possible to assess the daily risk of fire on Grande Terre in New Caledonia. We first describe how we built the model to assess the risk of fire ignition. Then we explain how our web application works. Finally, we discuss the value of such an application.

\section{ESTIMATING FIRE RISK IN NEW CALEDONIA}

The INC project analysed interactions between ecosystems, human practices (FAO, 2007), climate (André and Mangeas, 2012) and fire, and developed a geographic information system (GIS) to simultaneously monitor these components in space and over time (http://www.espace.ird.fr/index.php/projets-71/49projets/projets-en-cours/77-incendie-et-biodiversite-des-ecosystemes-de-nouvelles-caledonie). Analyses allowed the creation of a dynamic non-linear model to assess the risk of wildfire for New Caledonian ecosystems. The risk is estimated daily for each point of the main-land (Grande Terre). Using this model, the risks for biodiversity and soil erosion are computed.

Fire risk is the probability of a fire occurring in a specific location and under specific conditions, with its expected impact on the objects it affects (Bachmann and Allgower, 2001). Thus, the quantitative fire risk for a particular geographic region and time period can be calculated by combining two main components: burn probability and associated fire behaviour gauged by its intensity and spread rate (Finney, 2005). The potential damage from a fire can also be taken into account in the calculation of the risk (Chuvieco et al., 2010) (Mangeas et al., 2013).

The model to estimate fire risk is integrated, spatially explicit, and dynamic. It is based on analyses of environmental, climatic, and social conditions and provides starting probabilities for each geographical location and every day. All the input layers of the model were standardised in a grid raster with a $300 \mathrm{~m}$ resolution. The uncertainty of remote sensing data $(250 \mathrm{~m}$ for Normalized Difference Vegetation Index, $1 \mathrm{~km}$ for burned areas) and climate data (6 meteorological indices on 16 zones on the main-land) is integrated in this $300 \mathrm{~m}$ resolution, as well as the finer resolution of other layers such as roads, tribes and villages for instance. There are several variables for each of the three components (environmental, climatic and social) of the model. Each of these variables can have different states, and each of these states is associated with a probability of fire starting. The combination of these probabilities according to the theory of Bayesian networks provides the model to assess fire risks. Probability values are high (close to 1) when conditions are favourable for a fire to start, and low (close to 0 ) when adverse conditions prevail. In fact, the model does not give the probability of a fire starting, which depends on whether someone actually starts a fire, but rather a wide range of potentially favourable places for a fire to start. We nevertheless use the expression "ignition probability" to refer to what is actually an "assessment of the difficulty for a fire starting".

Every ignition pixel induces an area on which the fire spreads; therefore a propagation model was computed with fire characteristics (intensity, spread speed) assessed with combustibility, weather conditions (wind) and 
topography. The simulation was done depending on a scenario favourable to a fast spreading of fire on a maximum of eight hours. The risk of impact on plant biodiversity is computed by coupling the ignition model with the propagation model and a map of plant species. In addition, the amount of soil lost due to the passage of a fire is estimated, which helps assess the risk of erosion associated with the risk of fire.

\section{A WEB-BASED APPLICATION FOR A DAILY ASSESSMENT OF THE RISK OF A FIRE STARTING}

\subsection{Motivation}

The goal of the application is to make the results of the three models (ignition risk, risk for biodiversity, and risk of erosion) available to a wide audience, including decision makers and everyone concerned with fires, by providing daily information obtained with the model. The ability to combine the new information obtained with scientific models, with other geographic data is an added advantage.

To achieve this objective, we propose a web-based application including a geographic information system (GIS). Access is simple since all the user needs is an Internet connection. Thanks to an automated process, data are updated and processed by the model daily. The application is designed for people who are not necessarily experts in geomatics and is thus intentionally simple and user-friendly. Dynamic maps were chosen as appropriate for this category of users.

Different technologies were used to build the application. Each technology plays a specific role in the process. In the following section, we describe each component and its function, how automation was possible, and finally, interactions between these technologies.

\subsection{Application components: remote data process}

Our application had to be able to implement the model built in the INC project and to make the results available on the Internet.

\section{A window on the world: the web server}

A web server is the basis of a web application. We used "Apache HTTP server" (http://httpd.apache.org/), commonly known as "Apache". We needed a programming language to manage actions on the server side, i.e. retrieving information from the client (the user's browser), telling the server to perform tasks such as calculation based on a scientific model, and returning additional information resulting from data processing to the client. For this purpose, we chose PHP (Hypertext Preprocessor - http://php.net/).

Statistical studies and building the model were mainly performed using R software (R Development Core Team, 2011). The final model is therefore available as an $\mathrm{R}$ script, and some data used for the development of the model are stored as $\mathrm{R}$ variables.

\section{Data storage and return}

Several types of data are needed by our model to estimate fire risk, including land occupation, soil loss and the weather indices computed by Météo-France. All these data are stored in different formats. Some are available as Geo-Tiff images, others as ASCII or CSV files; others are stored as R variables in R archive files. $\mathrm{R}$ software is able to collect data from these different sources. The model results are provided as PNG and Geo-Tiff images, or as text describing geographic features in GML (Geography Markup Language) format.

\section{Server-user exchanges}

The user has to send the parameters specifying which data the model should use. The use of the AJAX concept (Asynchronous JavaScript and XML) prevents the reload of a full page for each user request. This concept is based on several technologies including JavaScript and XML or JSON (JavaScript Object Notation). The user sends queries with parameters and receives a response from the server once the request has been processed. The answer leads to changes in some parts of the user's web page. For this purpose, we used the jQuery JavaScript library (http://jquery.com/).

\subsection{Application components: display on dynamic maps}

To make the application user-friendly, we chose to present results provided by the model on dynamic maps. The user can interact with the map, which improves the efficiency of the representation and of the use of the tool itself. We now describe how the dynamic map display was achieved. 


\section{Use of OGC standards}

OGC is an international organization in which governments, trade research, and non-profit structures collaborate to implement open standards related to services and geospatial content, as well as GIS data processing and sharing (http://www.opengeospatial.org/). For the description of geographic features, the GML specification was implemented by OGC. OGC has also produced a standard, called WMS (Web Map Service), for the use of geo-referenced images (http://www.opengeospatial.org/standards/wms). This standard specifies how to create a Web Map Server (WMS), i.e. a server that functions in accordance with WMS standards, how to store geo-referenced images, how to supply them to clients, and how to query the WMS server (Open Geospatial Consortium Inc., 2006). The WMS standards are used for an easy display of dynamic maps on a web page, particularly for the display of the base map on the dynamic map of our application.

We used the open-source free software GeoServer. This software implements a very efficient WMS and is the benchmark for implementation of other OGC standards such as WFS (Web Feature Service) and WCS (Web Coverage Service).

\section{Display of dynamic maps}

The map is displayed using the open-source free JavaScript API (Application Programming Interface), named OpenLayers (http://openlayers.org/). This API makes it possible to query a WMS map server that responds by sending the URL for geo-referenced images that match the query. The images are displayed in a specific part of the web page, creating a base map. The OpenLayers library can also display images superimposed on the base map and read descriptions of geographic features in GML files to display them on the map. Several layers can be stacked, making it possible to compare different entities and available data.

\section{Output files}

As mentioned above, $\mathrm{R}$ provides the model implementation results in the form of geo-referenced PNG or Geo-Tiff images, and GML files.

Images are built from an R RasterLayer object in the R raster library (Hijmans and Van Etten, 2012). This type of object is useful to handle geo-referenced raster data, as it can include several layers. When creating the PNG results image, the limits of the treated zone are saved in a file. This is necessary because a PNG image cannot contain geographic information, but the limits of the area covered by the image have to be specified to enable OpenLayers to correctly display the image in a particular area of the map.

GML files allow geographic data to be described in vector form. They are created from $\mathrm{R}$ objects SpatialPolygonsDataFrame for polygons and SpatialLinesDataFrame for lines, available in the sp library (Pebesma, 2004). Each of these objects contains a geographical description of each entity and the values (quantitative or qualitative) associated with it. A function from the rgdal library (Keitt et al., 2012) is used to save these types of objects in a GML file.

\subsection{Automation of the application}

The weather indices used to assess fire risk vary from day to day, so the indices need to be updated every day to provide a reliable estimate. Météo France has established an address where files containing daily weather indices are available. Each day, a Python script downloads the data to be stored on the server that hosts our application.

Since the calculation needed to estimate the fire risk can be time consuming, we decided to store some results on the server hosting the application, especially those for the last thirty days. When a new file containing the weather index is stored on the server, $\mathrm{R}$ is run to perform calculations on these new data. This means the user has instantaneously access to results for the last thirty days (the recent days are the most likely to be requested). On the other hand, for older days, the waiting time may be much longer by at least two minutes, because of the time needed to perform the calculations.

\subsection{Execution of the application}

Here we summarize the different steps of our application from the access to our page to the results displayed on the dynamic map. Figure 1 illustrates these steps.

When the page is loading, OpenLayers asks the WMS map server for geo-referenced images of the area of interest (1). After the server has responded, the map of the area is displayed on the page of our application along with a form to allow the user to choose a date. The user's choice is sent to the server hosting the 
application and the R calculation software (2). PHP receives the information, processes it and controls the R execution according to the parameters sent by the user (3). R checks whether the files corresponding to the parameters sent by the user already exist (4). Their existence usually depends on the proximity of the date to today's date. Generally, files exist for recent dates (the last thirty days) and for dates that have recently been requested. If files already exist, R simply creates files indicating where to find the results (8), and then PHP takes over. If files have to be created, a specific $\mathrm{R}$ process begins.

The meteorological, geological, soil, topographic, environmental or societal information needed for the model implementation is collected (5). The information is then processed with the model we built (6). The results of the model applied to these data are stored in spatial files, as either images or GML files (7). Additional files, usually text files, are created (8). They specify where to find the output files created as a result of the model application. When outputs are PNG image files, a file showing the area boundaries and characteristics of the built image is also recorded.

Then PHP gathers all the information needed to allow access to the results and their display on the client side, and sends this information (9) as the response to the initial request from the client. Finally, from the information received, OpenLayers accesses spatial files and displays their contents as an overlay on the map.

Apart from the use of our application by any user, meteorological data files are automatically created or updated twice a day (A). Also thanks to this automatic action, $\mathrm{R}$ runs the model with new data from the same day (B) to create files containing the model outputs (C). Other data, such as land occupation, also vary over time, but the changes take place more slowly than meteorological variations. Such data need to be regularly updated, but less frequently than meteorological data, and updates are manual. Data updates ensure the sustainability of the estimations.

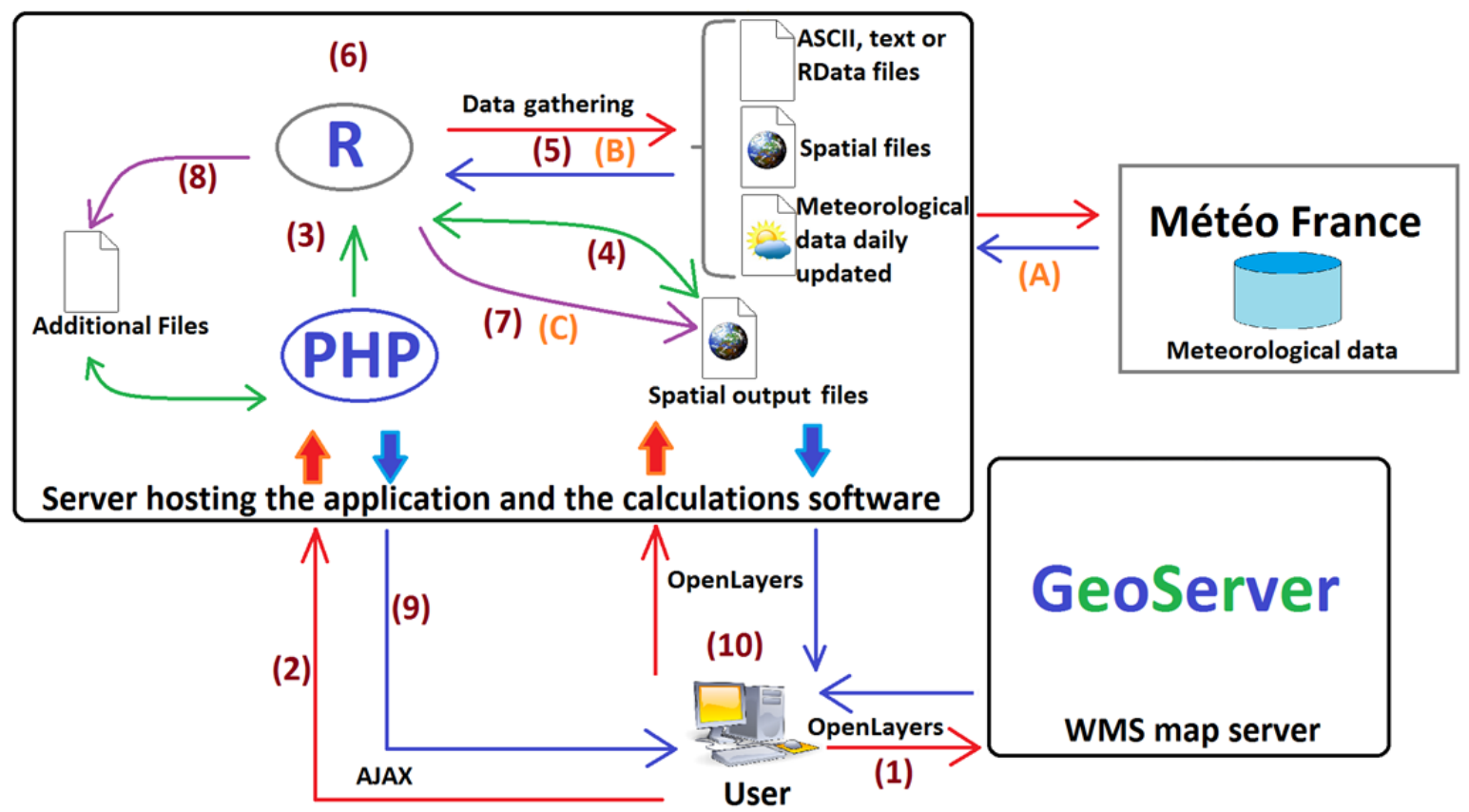

Figure 1. Interactions between technologies for the execution of the application that calculates the estimated fire risks in real time

The prototype of this application is available at: http://deployeur.univ-nc.nc/r/Feux_OpenLayers_PHP.html. Figure 2 is a screen shot from this prototype displaying an output map showing assessment of fire risk.

\section{ADVANTAGES AND POSSIBLE IMPROVEMENTS}

\subsection{Advantages of a web-based GIS}

Because the application is web based, it is widely accessible. This is thus a good way to highlight scientific research, especially for this particular project since a wide range of fields was explored and research data combined to achieve the project's goals. This implies demonstrating the value of the research to the general public. Users who want to know the risk of a fire on Grande Terre on a particular date do not have to install 
the calculation software with which the model was built, nor to access the data or the model used to estimate fire risk, nor to know how the model works by consulting the processing scripts.

Beyond the fact that anyone can benefit from this study, dialogue is facilitated between different stakeholders because, thanks to it, they have a common basis for discussion and decision making. Model results can be combined with other data to improve the discussion. A web-based application is particularly suitable for this purpose since data gathering is simplified.

Another advantage is that the model and the data can be updated. As the application and the data used for the implementation of the model are located on the server, updates take place only at this location. This greatly facilitates maintenance and access to a permanently updated application. Moreover, all stakeholders and decision-makers have access to these updates simultaneously with no effort required on their part, which would not be case if everyone had access to the model and to the data on his/her own computer. This represents a considerable time saving.

The model outputs are geographic data, but users do not have to be GIS experts to be able to view and understand these results, nor to dispose of GIS software to access the model assessments. The use of dynamic maps makes it possible to obtain a general view of the area of interest, but also to focus on a particular part of this area. All in all, the tool is a very flexible and user-friendly.

\subsection{Advantages of using OGC standards}

OGC has specified standards to make different systems interoperable, i.e. able to work with other existing or future systems without restricting access or implementation. A service that uses OGC standards is thus interoperable with other systems that also use OGC standards.

Describing geographic entities (model outputs) with GML makes it easy to display them on a dynamic map. Another application that needs to display results obtained from running the model could just as easily display results on a map.

The use of WMS specifications makes it easy to obtain a dynamic map. Comparison with other data is also simplified, for example the road network and the location of water holes. Anyone who needs to find the water holes located closest to potential fire sites has the right visual tool to take decisions. Geographic features that resemble houses, roads or water holes can be stored on a WFS (Web Feature Server), i.e. a server compliant with OGC WFS (Web Feature Service) specifications, for the storage, handling and collection of data concerning geographic features. Additional data, coupled with results from our model, will be possible under the WFS standards, which would make it easier to display and use them on the dynamic map. With such standards, the data with which a user wants to work can come from anywhere in the world, provided they are freely accessible.

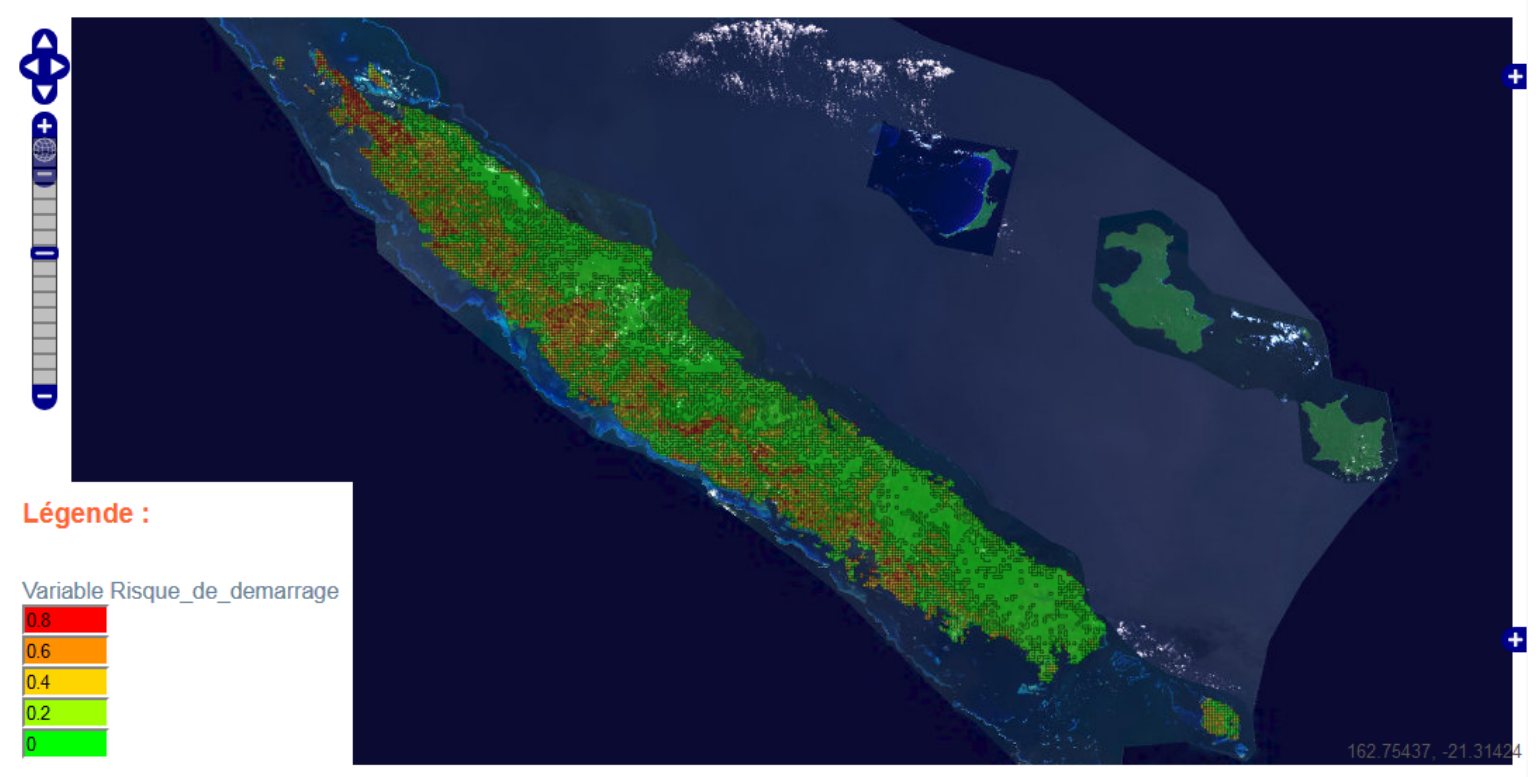

Figure 2. Map of fire risk assessment in New Caledonia on September $19^{\text {th }}, 2013$ 


\section{CONCLUSION}

In this paper, we presented a web application that allows an automated daily estimate of the risk of a fire starting in New Caledonia. This application highlights a four-year study conducted in different scientific domains as part of the INC project whose aim was to better understand fire starting and to build models of the risk of fire for biodiversity and erosion.

The application makes it possible for the user to benefit from the results of this study with nothing more than a simple Internet connection. The automatic (weather) data update ensures the application is sustainable and results are provided daily. Using the application, users can start a calculation on the server and subsequently retrieve the results, if they are not yet known, or simply access previously calculated results, in order to display them on their own web page. Results are standardized and geo-referenced so they can be easily embedded in a dynamic map.

The use of the standards developed by OGC, such as WMS, helps to make our application interoperable. The addition of external data is possible. Both these features help understand the fire phenomenon or take action based on the user's own observations once the model results are displayed. It is easy for analysts and decision makers to make decisions in their area of interest thanks to the superimposition of data from different sources.

Simplicity, intuitiveness and ease of use are very important assets since they mean stakeholders do not have to be GIS experts to obtain representations of scientific models. Anyone concerned by the fire issue in New Caledonia can use this tool to exchange ideas and discuss the most appropriate strategies to apply. Monitoring is now more efficient and will consequently help preserve biodiversity and the soil in New Caledonia.

\section{ACKNOWLEDGMENTS}

We acknowledge all partners in the INC project, particularly IRD and Météo France NC, without whom this automated application would not be possible.

\section{REFERENCES}

André, J., and M. Mangeas (2012). Modélisation de la probabilité de démarrage incendie sur la Grande-Terre : approche intégrée pluridisciplinaire. Technical report, Nouméa, 2012.

Bachmann, A., and B. Allgower (2001). A consistent wildland fire risk terminology is needed. Fire Management Today, 61.

Chuvieco, E., I. Aguado, M. Yebra, H. Nieto, J. Salas, M.P. Martin, L. Vilar, J. Martinez, S. Martin, P. Ibarra, J. de la Riva, J. Baeza, F. Rodriguez, J.R. Molina, M.A. Herrera, and R. Zamora (2010). Development of a framework for fire risk assessment using remote sensing and geographic information system technologies. Ecological Modelling, 221, 46-58.

FAO (2007). Fire management-global assessment 2006. FAO Foresty paper, 151.

Finney, M.A. (2005). The challenge of quantitative risk analysis for wildland fire. Forest Ecology and Management, 211, 97-108.

Hijmans, R.J., and J. Van Etten (2012). raster: Geographic analysis and modeling with raster data. http://CRAN.R-project.org/package=raster.

Keitt, T.H., R. Bivand, E. Pebesma, and B. Rowlingson (2012). rgdal: Bindings for the Geospatial Data Abstraction Library. http://CRAN.R-project.org/package=rgdal.

Mangeas, M., C. Gomez, T. Curt, T. Ibanez, J. Munzinger, P. Dumas, M. Despinoy, C. Hély (2013). Wildfire risk for main vegetation units in a biodiversity hotspot: modelling approach in New Caledonia, South Pacific. (in submission).

Open Geospatial Consortium Inc. (2006). OpenGIS Web Map Server Implementation Specification. Jeff de la Beaujardiere, 15 March 2006.

Pebesma, E.J. (2004). Multivariable geostatistics in S: the gstat package. Computers \& Geosciences, 30, 683691.

R Development Core Team (2011). R: A language and environment for. Vienne: R Foundation for Statistical Computing, Vienna, Austria. 